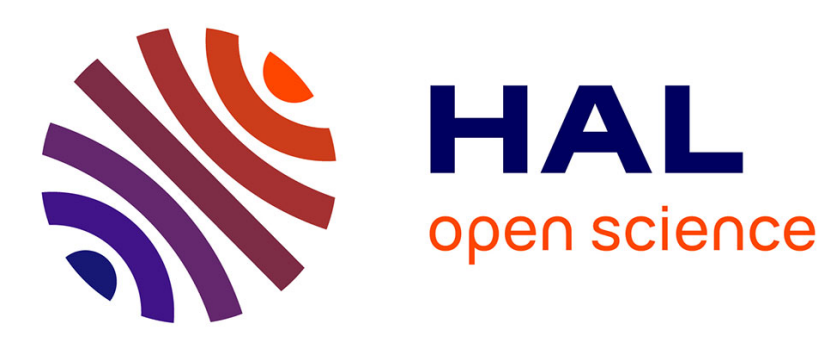

\title{
Diffraction of long period Rayleigh waves effects of mode coupling by a slab: effects of mode coupling
}

Philippe Lognonné, Eric Clévédé

\section{To cite this version:}

Philippe Lognonné, Eric Clévédé. Diffraction of long period Rayleigh waves effects of mode coupling by a slab: effects of mode coupling. Geophysical Research Letters, 1997, 24 (9), pp.1035-1038. 10.1029/97GL00948 . insu-01390093

\section{HAL Id: insu-01390093 \\ https://hal-insu.archives-ouvertes.fr/insu-01390093}

Submitted on 31 Oct 2016

HAL is a multi-disciplinary open access archive for the deposit and dissemination of scientific research documents, whether they are published or not. The documents may come from teaching and research institutions in France or abroad, or from public or private research centers.
L'archive ouverte pluridisciplinaire HAL, est destinée au dépôt et à la diffusion de documents scientifiques de niveau recherche, publiés ou non, émanant des établissements d'enseignement et de recherche français ou étrangers, des laboratoires publics ou privés. 


\title{
Diffraction of long period Rayleigh waves by a slab: effects of mode coupling
}

\author{
Philippe Lognonné and Eric Clévédé \\ Dept. de Sismologie, URA 195, Institut de Physique du Globe de Paris, France
}

\begin{abstract}
We compute seismograms of the fundamental Rayleigh waves propagating through a slab structure, with either a lateral variation in seismic velocities, or in attenuation. At periods of $100 \mathrm{sec}$, we show that the phase delay is strongly reduced by the surface waves Fresnel zone, and that coupling must be considered far along the dispersion branch, up to at least $\ell \pm 25$. Limiting the coupling to fewer modes produces a signal associated to a ghost structure at the antipode of the slab. We also show that the amplitude perturbations produced by the diffraction and the attenuation of the slab are comparable in size.

Future waveform studies, especially those associated to global waveform inversions, must then carefully consider these effects.
\end{abstract}

\section{Introduction}

Tomographic models provide global maps of the Earth smooth 3D elastic [e.g. Montagner \& Tanimoto, 1991, Li $\mathcal{G}$ Romanowicz, 1996] and anelastic [Romanowicz, 1995] structure. However, these models cannot resolve the mantle small scale lateral variations. This is partially due to the insufficient coverage of the global networks, but we can also consider that the theories used for such inversions have reached their limit. Indeed, most of the inversions are done with asymptotic (e.g. Romanowicz, [1987]) or WJKB theories (e.g. Tromp \& Dahlen, [1992]) which are valid only when the scale of heterogeneities are large with respect to the wavelength. Higher resolution is then achieved with higher frequency data. For surface waves this however reduces the signal to noise ratio at below periods of $30 \mathrm{sec}$ and complicates the source model.

Also many inversions deal only with phase perturbations. The stationarity of the Fermat principle reduces the impact of modeling and data errors but also reduces the contributions of the Earth's small scale structure, when considered as a perturbation to the smooth 3D structure. It is well known for example that odd structure produces a second order coupling effect for eigenfrequencies, but a first order effect for amplitudes.

Amplitude information and a better modeling of the

Copyright 1997 by the American Geophysical Union.

Paper number 97GL00948.

0094-8534/97/97GL-00948\$05.00 seismic waves, can then be considered as mandatory for the elaboration of a new generation of tomographic model of the Earth with increased resolution. In this paper we test the efficiency of the higher order perturbation theory, [Lognonné, 1991] , to compute waveforms propagating through small scale structures.

\section{Model and Theory}

We use a slab model, superimposed on an elliptical Earth (Figure 1). The slab has first a $2 \%$ increase of shear and bulk moduli, from the surface to the CMB. In a second test, it has a $20 \%$ increase of shear and bulk quality coefficient Q. Even if not fully realistic, this is typicaly of the size of some of Earth's short scale structures.

Interaction terms are computed with a spectral method [Lognonné \& Romanowicz, 1990]. Seismograms are done as an aspherical normal modes summation, and can be expressed for a step source at $t=0$ as [Clévédé \& Lognonné, 1996]

$$
s(t)=\sum_{K} \Re e\left(e^{i \sigma_{K} t} A_{K}(t)\right),
$$

where $\sigma_{K}$ is the spherical carrier frequency of the multiplet $K$ and $A_{K}(t)$ is the modulation function of the mode, defined as

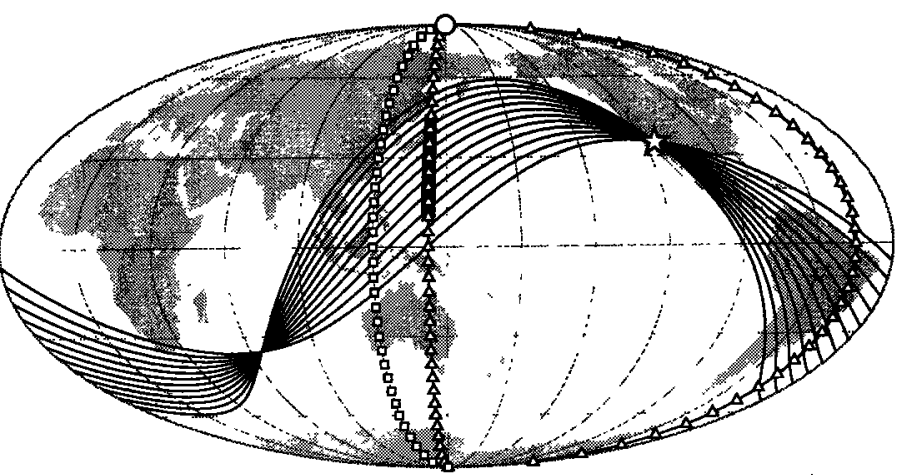

Figure 1. Map showing the slab structure which extends from $140 \mathrm{E}$ to $145 \mathrm{E}$ in longitude, and from $10 \mathrm{~N}$ to $45 \mathrm{~N}$ in latitude. The isotropic source is located at the North Pole in the first geometry, and triangles show the position of the receivers, covering the full meridians $142.5 \mathrm{E}$ and $37.5 \mathrm{~W}$, and a circle shows the position of the source. In the second geometry, the isotropic source is located at $35 \mathrm{~N}$ of latitude and $115 \mathrm{~W}$ of longitude and shown by a star, and squares show the position of the receivers, covering the full $120 \mathrm{E}$ meridian. 


$$
A_{K}(t)=\sum_{k \in K} r_{k} s_{k} e^{2\left(\sigma_{k}-\sigma_{K}\right) t} .
$$

Here $k$ represents one of the singlets of $K$, and $\sigma_{k}, r_{k}, s_{k}$ are the eigenfrequency and the receiver and source amplitudes respectively. Practically, the modulation function $A_{K}(t)$ is first computed with a 30 minutes time step by using the amplitudes of the singlets at both source and receiver. It is then re-sampled to the seismogram sampling time (here $10 \mathrm{sec}$ ) using spline interpolations. The singlet amplitudes for the source and all receivers are tabulated, which allows the final seismograms computations to be done on a workstation.

\section{Slab test with a velocity perturbation}

A first test with the elastic shear and bulk perturbation was performed for the fundamental Rayleigh wave up to $10 \mathrm{mHz}$. In this test, the coupling along the fundamental branch up to a coupling coefficient of $\epsilon=10^{-3}$ was taken, corresponding to coupling of $\ell \pm 12$ close to $100 \mathrm{sec}$. Figure 1 shows the receivers and source geometry, all along the symmetry meridian of the slab (142.5 E). The accuracy was however not sufficient: we found that the R2 wave packets were perturbed by a "ghost" structure at the slab antipode, resulting from the coupling truncation. The extreme case is the isolated multiplet approximation, where only the symmetric structure, i.e. two equal structures at the slab location and its antipode, are modeled. For our slab model, the coupling strength coefficients between multiplets decrease slowly. For $\ell=90$ for example, the strength is $10^{-2}$ for the $\ell \pm 1$ only and reaches $10^{-4}$ for $\ell \pm 25$. Only for smooth models, with red spectrum, does the truncation of coupling terms have a limited effect as shown for example by Um \& Dahlen, [1991], but not for a model with small scale structures and a much whiter spectrum (the slab's spectrum has cutoffs at $s=5$ and $s=35$ ). Indeed, a second modeling, done up to $\epsilon=10^{-4}$, corresponding to coupling up to $\ell \pm 24$ strongly reduces the signal from the ghost structure. Figure [2a] shows the residual between the seismograms computed with the slab structure, and those without. The comparison between the two type of seismograms, as well as the residual for the receiver located at the end of the slab (latitude 10N), is shown on Figure [2b]. Note that neither R1 and R2 detect the slab structure before passing through it. Note also the focalisation and de-focalisation of the residual, which is related to the destructive interaction of the self-coupling part and the coupled part of the seismograms. As for 2-D studies [Friederich et al., 1993], both the (first order) coupling and the higher orders have impact on the waveform by reducing non-causal perturbations.

At the end of the slab structure, $8-9.5 \mathrm{mHz}$ band filtered seismograms have a time delay of about $-5.5 \mathrm{sec}$, compared to the only elliptical Earth. This is much less than the time shift computed by ray theory, about $-10.5 \mathrm{sec}$. Tests with different coupling have shown dif-
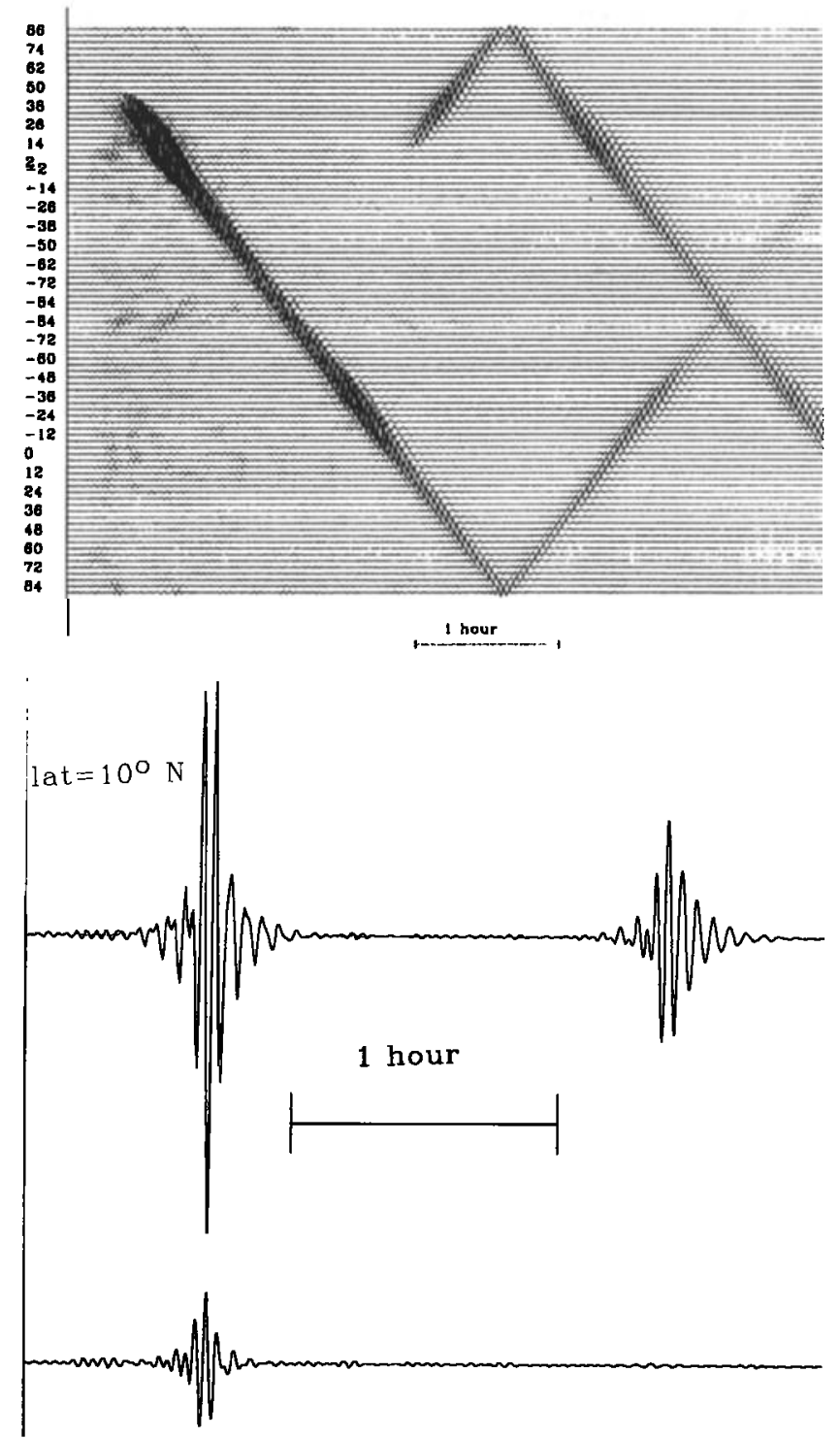

Figure 2. Top (a): Residual between the seismogram obtained with and without the slab for a coupling up to $\epsilon=10^{-4}$ and the first source/receiver geometry. Here and in Figures 4 \& 5 , seismograms were bandpass filtered with a cosine filter between $4-5 \mathrm{mHz}$ and $8-9 \mathrm{mHz}$. Amplitudes are normalized to the maximum residual observed at the end of the slab and which reaches a maximum of $35 \%$ of the waveform (see b). The residuals express both the phase and amplitude perturbations. Bottom (b) shows the amplitude of the residual with respect to a seismogram computed in the Elliptical model, recorded at the end of the slab (10 N of latitude). The top trace shows the elliptical and slab traces superimposed, the latter being smaller by $17 \%$.

ferences of up to $0.5 \mathrm{sec}$ between coupling with $\ell \pm 10$ and $\ell \pm 20$, and up to $0.15 \mathrm{sec}$ between the latter and $\ell \pm 30$, so that this low time delay is probably not due to insufficient coupling. This smaller shift is however probably due to the surface wave Fresnel zone, (see e.g. Yomogida, [1992]), which is implicitely modelled by the mode summation (see e.g. Li \& Romanowicz, [1986], in the case of normal modes summation for body waves). 


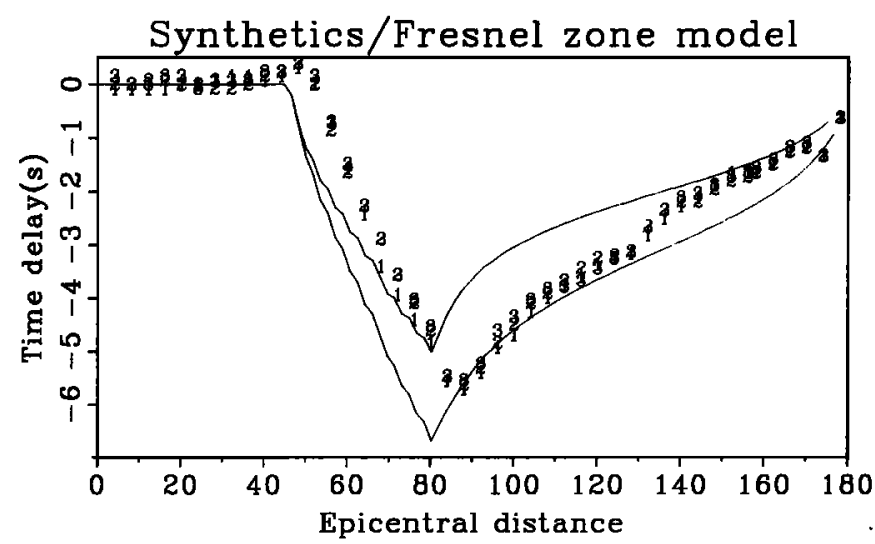

Figure 3. Comparison of the Fresnel zone's time shift with the shift between synthetics with and without slab. Seismograms were filtered in the band $8-9.5$ by a cosine bandpass filter, and the delay was found by crosscorrelation (symbol 1,2,3 for coupling at $\ell \pm 10,20,30$ respectivelly). Up to 120 degrees the shift is well explained by a cosine Fresnel zone (bottom line). At greater distances the shift is better approximated by a boxcar Fresnel zone (upper line).

More precisely, let us consider a meridien path, where both the source and receiver are located, and which is taken as the origin of longitude. The limit of the Fresnel zone will correspond to the points on the sphere, with latitude $\theta$, and longitude $\Phi(\theta)$, for which the angular distances $\Delta_{1}$ and $\Delta_{2}$ to the source and receiver respectivelly are such that:

$$
\Delta_{1}+\Delta_{2}=\Delta+\frac{\lambda}{4 a}
$$

where $\Delta$ is the distance between source and receiver, $\lambda$ the surface waves's wavelength and $a$ the Earth's radius. We can then approximate the time delay as

$$
\Delta t=\frac{\Delta t_{0}}{L} \int d \theta f\left(\frac{d}{\Phi(\theta)}\right)
$$

where $d$ and $L$ are the angular width and length of the slab respectively, $\Delta t_{0}$ the high frequency delay time and $f$ a taper function. For the synthetics of Figure 2, Figure 3 shows the comparison between the observed time shift and the time shift expressed by relation (4) with a cosine and boxcar function $f$. For a receiver in the slab, about $1 \mathrm{sec}$ of difference remains, probably due to the limits of our simple model of Fresnel zone. The fit is much better for a receiver outside: the Fresnel zone is low pass filtering the structure with a cutoff at $s=20$ or less for the receivers in the Southern hemisphere, which reduces the effect of truncation.

For the second geometry of Figure [1], coupling up to $\ell \pm 24$ is still insufficient to fully model the transmitted wave Figure [4]. A ghost signal still remains before the $\mathrm{R} 1$ and $\mathrm{R} 2$ wave packets with amplitudes of 10 to $20 \%$ of the main residual.

Our example practically shows the importance of coupling terms in any computation with short scale structure, and especially for future work aiming at resolving

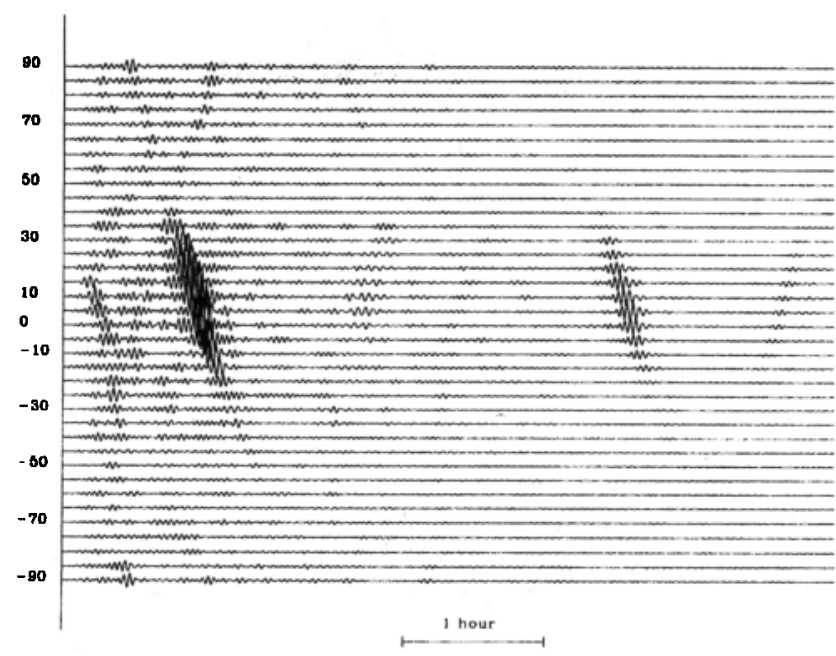

Figure 4. Same as for Figure 2a, but for the second source/path geometry. Strong non causal residual, associated to the insufficient coupling, appear before the main arrival, but also at the position of the $R 2$ wavepacket.

short scale structure models, for which the spectrum may not decrease so sharply as for the $s=2-8$ angular orders. The coupling between about 10000 singlets at $\ell=90$ need to be computed, which will make the variational method very heavy. On the contrary, perturbation theory with spectral methods will be much more efficient.

\section{Slab test with a perturbation in attenuation}

We put now a Q-shear and Q-bulk perturbation of 20 $\%$ in the slab and compute fundamental mode synthetics up to $10 \mathrm{mHz}$, taking coupling up to $\ell \pm 24$. The

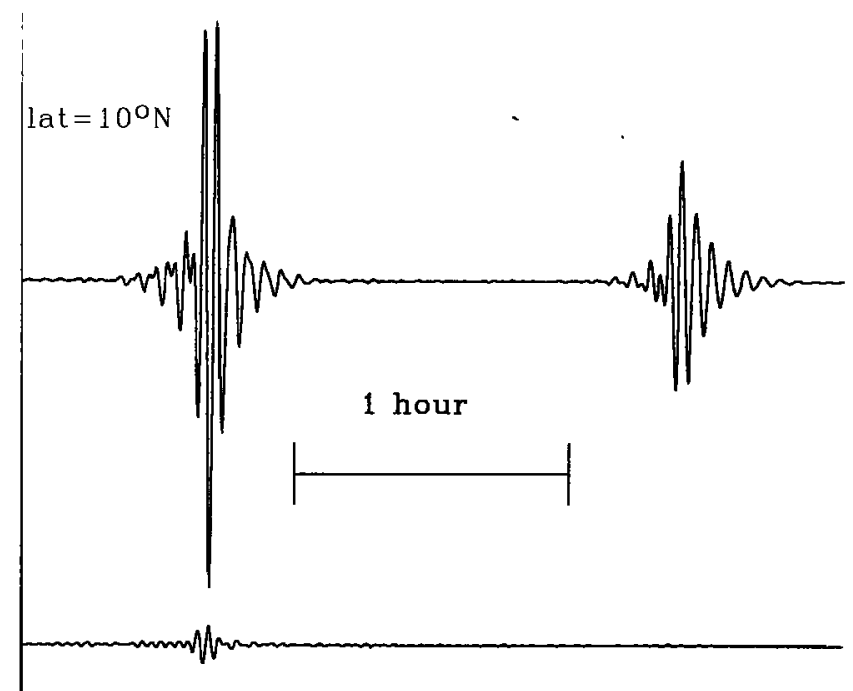

Figure 5. Same as for Figure $2 b$, but with a slab having a $Q$ lateral variation of $20 \%$. Both the seismogram and its residual with respect to a seismogram without slab are recorded at $10 \mathrm{~N}$ of latitude, at the end of the slab. The top trace shows the elliptical and slab trace superimposed, the latter being smaller by $2.4 \%$. 
geometry is for the north-south path of Figure 1 . We show here results assuming a causal lateral variations in $Q$ with a logarithmic dispersion with a reference period of $1 \mathrm{sec}$ [Liu et al., 1976]:

$$
\delta\left(\frac{\mu}{Q}\right)\left(\frac{2}{\pi} \operatorname{Ln}\left(\frac{\omega}{\omega_{\text {ref }}}\right)+i\right)
$$

The residuals, compared to the previous elastic perturbation, have very small amplitudes, as shown on Figure [5]. Here, the low attenuating slab decreases the amplitude of the residuals. For example, at $8.5 \mathrm{mHz}$, the complex perturbation in seismic velocity reaches $0.5 \%$ in amplitude and produces, by diffraction, a reduction of the order of $5 \%$ of the seismogram amplitude, assuming that the amplitude of the diffracted wave can be scaled from the first test results. On the other hand, the perturbation in attenuation is smoothed by the Fresnel zone, and produces a positive amplitude perturbation of $2.7 \%$. The superposition of these two effects explains then well the $2.4 \%$ amplitude reduction observed on the synthetics, as well as a time delay of $-1.45 \mathrm{sec}$. The diffraction and attenuation of small and attenuating structures produce signals comparable in amplitudes, which will require the use of the complete waveform for separation.

\section{Conclusion}

We have shown that the effect of small scale heterogeneities in both elastic and anelastic structure can be computed using higher order perturbation theory. The coupling terms are slowly converging on the waveform, and these computations require to couple terms far along the dispersion branch. Neglecting these terms induces ghost arrivals, associated with a virtual structure at the antipode of the heterogeneity. At $T>100 \mathrm{sec}$, the synthetics delay times are much less than those obtained by the path average approximation. Neglecting these effects leads to underestimate the amplitude of small scale heterogeneities of the Earth. Extracting from seismograms information about the small scale elastic and anelastic structure of the Earth will require careful modeling of the coupling effects.
Acknowledgments. Thanks to B. Romanowicz for a constructive review. This work was done with the CM5128 computer of the "Centre National de Calcul Paralelle en Sciences de la Terre" and with support of the I.N.S.U. ATP Tomographie. I.P.G.P contribution 1463.

\section{References}

Clévédé E. and P. Lognonné, Fréchet derivative of coupled seismograms with respect to an rotating, anelastic, aspherical Earth, Geophy. J. Int., 124, 456-482, 1996. Corrigendum in Geophy. J. Int., 126, 903, 1996.

Friederich, W., E. Wielandt and S. Stange, Multiple forward scattering of surface waves:comparison with exact solution and Born single-scattering methods, Geophys. J. Int., 112, 264-275, 1993.

$\mathrm{Li}, \mathrm{X}$.-D. and B. Romanowicz, Global shear velocity model developed using non-linear asymptotic coupling theory, $J$. Geophys. Res., 101, 22245-22272, 1996.

Liu, H.P., Anderson, D.L. \& H. Kanamori, Velocity dispersion due to anelasticity: implications for seismology and mantle composition, Geophys. J. R. Astr. Soc., 47, 41-58, 1976.

Lognonné P., Normal modes and Seismograms of an anelastic rotative Earth., J. Geophys. Res., 96, 20309-20319, 1991.

Lognonné, P. and B. Romanowicz, Fully coupled Earth's vibrations: the spectral method, Geophys. J. Int., 102, 365-395, 1990.

Montagner, J.P. and T. Tanimoto, Global upper mantle tomography of seismic velocities and anisotropies, J. Geophys. Res., 96, 20337-20351, 1991.

Romanowicz, B., Multiplet-multiplet coupling due to lateral heterogeneities: asymptotic effect on the amplitude and frequency of Earth's normal modes, Geophys. J. R. Astr. Soc., 90, 75-100, 1987.

Romanowicz, B., A global tomographic model of shear attenuation in the upper mantle, J. Geophys. Res., 100, 12375-12394, 1995.

Tromp, J. and F.A. Dahlen, Variational principles for surface wave propagation on a laterally heterogeneous EarthI. Time domain JWKB theory, Geophys. J. Int, 109, 581$598,1992$.

Yomogida, K., Fresnel zone inversion for lateral heterogeneities in the Earth. Pageoph, 138, 391-406, 1992.

Philippe Lognonné and Eric Clévédé, I.P.G.P, Département de Sismologie, URA 195 CNRS, 4, place Jussieu, 75252 PARIS cedex 05, FRANCE

(received July 1, 1996; revised February 17, 1997; accepted March 5, 1997.) 